\title{
Interstitial Lung Disease and Diffuse Alveolar Hemorrhage, the Two Key Pulmonary Manifestations in Microscopic Polyangiitis
}

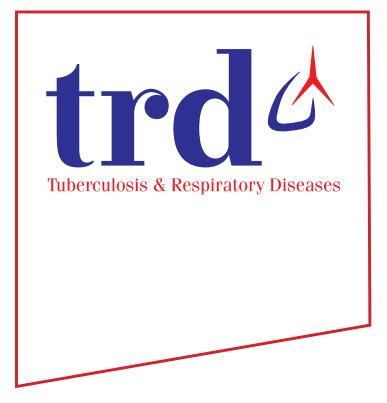

\author{
Min Jung Kim, M.D. (ii) and Kichul Shin, M.D., Ph.D. \\ Division of Rheumatology, Department of Internal Medicine, Seoul Metropolitan Government-Seoul National Boramae \\ Medical Center, Seoul, Republic of Korea
}

\begin{abstract}
Microscopic polyangiitis (MPA) is an antineutrophil cytoplasmic antibody (ANCA)-associated necrotizing vasculitis, which mainly affects small vessels in various organs, especially the lungs. The two key pulmonary manifestations, interstitial lung disease (ILD) and diffuse alveolar hemorrhage (DAH), increase the morbidity and death rate of patients with MPA. ILD is more common in MPA than in other ANCA-associated vasculitis subsets and is primarily associated with myeloperoxidase-ANCA. Unlike alveolar hemorrhage due to pulmonary capillaritis, ILD can initially manifest as isolated pulmonary fibrosis. Of note, its most frequent radiographic pattern is the usual interstitial pneumonia pattern, similar to the characteristic pattern seen in idiopathic pulmonary fibrosis. In this review we present the pathogenesis, clinical manifestations, and radiographic and histopathologic features of ILD and DAH in MPA. We also briefly summarize the outcome and therapeutic options for the two conditions.
\end{abstract}

Keywords: Microscopic Polyangiitis; Interstitial Lung Disease; Diffuse Alveolar Hemorrhage; ANCA; Myeloperoxidase

\section{Introduction}

Antineutrophil cytoplasmic antibody (ANCA)-associated vasculitis (AAV) is a systemic necrotizing vasculitis that predominantly affects small vessels in the upper and lower respiratory tract, kidneys, skin, and peripheral nerves. AAV includes microscopic polyangiitis (MPA), granulomatosis with polyangiitis (GPA), and eosinophilic granulomatosis with

\footnotetext{
Address for correspondence: Kichul Shin, M.D., Ph.D.

Division of Rheumatology, Department of Internal Medicine, Seoul Metropolitan Government-Seoul National Boramae Medical Center, 20 Boramae-ro-5-gil, Dongjak-gu, Seoul 07061, Republic of Korea Phone: 82-2-870-3204, Fax: 82-2-870-3866

E-mail: kideb1@snu.ac.kr

Received: Apr. 16, 2021

Revised: May. 27, 2021

Accepted: Jul. 7, 2021

Published online: Aug. 20, 2021

(c) It is identical to the Creative Commons Attribution Non-Commercial License (http://creativecommons.org/licenses/by-nc/4.0/).
}

polyangiitis (EGPA). Each entity is diagnosed by means of its clinical phenotype, involved organ systems, biopsy characteristics, and type of the ANCA.

There are currently no diagnostic tests or reliable biomarkers for AAV. In 1990, the American College of Rheumatology (ACR) developed the classification criteria for vasculitis, including GPA and EGPA ${ }^{1}$. In addition, disease definitions for vasculitis were established in 1994 by the Chapel Hill Consensus Conference (CHCC) and were updated in $2012^{2}$. The CHCC definitions are often used in clinical studies to recruit patients with AAV. According to the CHCC definition, MPA presents as a necrotizing vasculitis, with few or no immune deposits, predominantly affecting small vessels. Necrotizing glomerulonephritis is commonly seen, and pulmonary capillaritis often occurs, but without granulomatous inflammation. Importantly, the ACR criteria and CHCC definitions are not used for diagnosis. In clinical practice, the diagnosis of $\mathrm{AAV}$ is based on clinical manifestations, ANCA testing, pathology of tissue biopsies, and imaging data.

MPA is associated with myeloperoxidase (MPO)-ANCA and less frequently with proteinase 3 (PR3)-ANCA. Compared to other types of AAV, patients with MPA are typically older and have higher death rates, but a lower relapse rate $\mathrm{e}^{3,4}$. MPA affects the small vessels in the kidneys, resulting in impaired 
renal function caused by progressive, focal necrotizing glomerulonephritis. The lungs are also involved in a substantial proportion of the patients with MPA, which ranges from 25\% to $55 \%{ }^{5}$. Depending upon the type of AAV, patients with AAV show specific pulmonary manifestations (Table 1). Although diffuse alveolar hemorrhage (DAH) is the most frequent and life-threatening pulmonary complication of $\mathrm{MPA}^{6}$, there has been an increase in reports on the incidence of interstitial lung disease (ILD) in $\mathrm{MPA}^{7-9}$. In some cases, ILD may lead to significant morbidity and death rates in patients with MPA, yet it is unknown whether the development of lung fibrosis is directly related to vasculitis ${ }^{7,10}$. In this review we present information for a better understanding of the two significant pulmonary manifestations of MPA, focusing on the clinical characteristics, radiographic findings, and pathogenic mechanisms of ILD in MPA.

\section{Interstitial Lung Disease}

\section{Epidemiology and clinical manifestations}

ILD with AAV is usually reported in patients older than 65 years, with a higher incidence in males $(60 \%-65 \%)^{11}$. It is mostly observed in MPA ( $7 \%$ to $43 \%$ of patients), but on rare occasions, it is also seen in other types of AAV (23\% in GPA, very rare in EGPA) $)^{7,8}$. In ANCA serology, ILD is primarily associated with MPO-ANCA $(46 \%-71 \%)$, whereas some cases $(0 \%-29 \%)$ are positive for PR3-ANCA ${ }^{7,9,12}$. In contrast, other pulmonary manifestations of $\mathrm{AAV}$, such as nodules or pulmonary infiltrates, are equally associated with both MPO- and PR3-ANCA ${ }^{13}$. The prevalence of ILD in AAV is higher in the Japanese population than in Caucasians ${ }^{14}$ because of the higher prevalence of MPA and MPO-ANCA in Japan, and the possible genetic predisposition of the Japanese to $\operatorname{ILD}^{15}$. However, the prevalence of ILD in patients with MPA appears to be similar worldwide. Previous studies also identified pulmonary manifestations in patients with MPA in Korea. A single-center cohort study found that $20.4 \%$ of patients with AAV had ILD at diagnosis ${ }^{16}$. Ahn et al. ${ }^{17}$ reported that ILD was present in $23.6 \%$ of Korean patients with MPA, with usual interstitial pneumonia (UIP) being the most frequent (84.6\%) feature.

The main symptoms of ILD in MPA are usually nonspecific and include progressive dyspnea on exertion and/or nonproductive cough, similar to those of idiopathic pulmonary fibrosis (IPF). Pulmonary symptoms can be masked by the robust constitutional and extra-pulmonary manifestations of vasculitis, which include fever, weight loss, arthralgia/myalgia, skin rash, or paresthesia ${ }^{5}$. Interestingly, MPA patients with ILD have less vasculitis activity, lower erythrocyte sedimentation rate, and less frequent DAH, peripheral neuropathy, and glomerulonephritis than do those without ILD ${ }^{18}$.

\section{ANCA-positive idiopathic interstitial pneumonia}

ILD occurs in most patients with AAV at the same time $(36 \%-67 \%)$ as or before the onset of vasculitis $(14 \%-85 \%)^{19}$. It is not common for the onset of AAV to precede the diagnosis of ILD (8\%-21\%). In particular, ILD can initially manifest as ANCA-positive idiopathic interstitial pneumonia (IIP), and overt vasculitis can develop months to years later ${ }^{11}$. The prevalence of MPO-ANCA in patients with IIP ranges from $4 \%$ to

Table 1. Airway and pulmonary manifestations in ANCA-associated vasculitis ${ }^{11,19-22}$

\begin{tabular}{|c|c|c|c|}
\hline & $\begin{array}{c}\text { Microscopic polyangiitis } \\
(\%)\end{array}$ & $\begin{array}{l}\text { Granulomatosis with } \\
\text { polyangiitis (\%) }\end{array}$ & $\begin{array}{c}\text { Eosinophilic granulomatosis } \\
\text { with polyangiitis }(\%)\end{array}$ \\
\hline \multicolumn{4}{|l|}{ Upper airway manifestations } \\
\hline Sinusitis & Rare & 61 & $14-73$ \\
\hline Nasal mucosa ulcers/bleeding & Rare & Up to 70 & Rare \\
\hline Saddle nose & Rare & $20-50$ & Rare \\
\hline \multicolumn{4}{|l|}{ Lower airway manifestations } \\
\hline Asthma & 5 & 8 & $95-100$ \\
\hline Tracheal stricture and stenosis & Rare & 15 & Rare \\
\hline Bronchiectasis & $16-32$ & $13-20$ & $15-20$ \\
\hline \multicolumn{4}{|l|}{ Pulmonary manifestations } \\
\hline Lung nodules & $7-30$ & $30-89$ & $11-89$ \\
\hline Diffuse alveolar hemorrhage & $10-55$ & $5-30$ & $3-8$ \\
\hline Lung fibrosis/interstitial lung disease & $32-45$ & 23 & Rare \\
\hline \multicolumn{4}{|l|}{ Pleural manifestations } \\
\hline Pleural effusion & $22-27$ & $12-20$ & $12-22$ \\
\hline
\end{tabular}


$35 \%$, whereas PR3-ANCA is rare $(2 \%-4 \%)^{19}$. Patients with ANCA-positive IIP usually present with symptoms of shortness of breath or cough, whereas hemoptysis and constitutional symptoms are less frequent. The risk of developing vasculitis, mainly MPA, is mostly limited to subjects with MPO-ANCA, and $7 \%$ to $40 \%$ of the patients with IIP plus MPO-ANCA are diagnosed with MPA during follow-up. Previous studies suggested that IIP patients with more eosinophils in bronchoalveolar lavage (BAL) fluid, higher ANCA titers, and more lowattenuation areas in the chest in computed tomography (CT) had an increased risk of developing $\mathrm{MPA}^{23}$. In addition, the presence of rheumatoid factor could be a predictor of the future development of $\mathrm{MPA}^{24}$.

\section{Pathogenesis}

Several pathogenic mechanisms may contribute to the development of ILD in MPA. First, pulmonary fibrosis may result from repeated episodes of alveolar hemorrhage caused by capillaritis, and there is evidence that cell-free hemoglobin induces alveolar epithelial injury mediated by the redox transition of hemoglobin to higher oxidation states ${ }^{25}$. Previous studies have reported the histological evidence of acute or chronic alveolar hemorrhage in lung specimens and of increased hemosiderin-laden macrophages in the BAL fluid of $\mathrm{AAV}$ patients before they present with overt $\mathrm{DAH}^{26,27}$.

It has also been suggested that MPO-ANCA may play a pivotal role in the pathogenesis of pulmonary fibrosis, considering the predominance of MPO-ANCA in this group of patients. By activating MPO, oxidative stress and hypochlorous acid could also trigger fibroblast activation and subsequent lung fibrosis in MPA in vitro ${ }^{28}$. In addition, MPO-ANCA activates neutrophils and may directly contribute to lung-tissue damage by the proteolytic enzymes released from ANCA-activated neutrophils ${ }^{29}$. A previous study showed that neutrophil elastase, one of the proteolytic enzymes, could induce pulmonary fibrosis in experimental animal models ${ }^{30}$. There is also an increased proportion of neutrophils in the BAL fluid as well as in the lung tissue of AAV-ILD patients ${ }^{31}$. Thus, repeated episodes of inflammatory alveolar injury can lead to a reactive fibrotic state.

Tissue damage induced by eosinophils and neutrophil extracellular traps (NETs) could also play a role in the development of ILD ${ }^{11}$. Extensive tissue eosinophilia has been reported in AAV patients with marked interstitial fibrosis ${ }^{26}$. Moreover, BAL eosinophilia has been suggested as a marker of progressive pulmonary fibrosis ${ }^{32}$. NETs retain MPO or PR3, thus help break immune tolerance, and induce autoantibody formation. The induction of NETs by activated neutrophils correlates with MPO-ANCA affinity in MPA patients ${ }^{33}$. Other factors that are related to the development of ILD in MPA include smoking and chronic lung parenchyma ischemia ${ }^{11}$.

Finally, the association of $M U C 5 B$ promoter polymorphism with ILD in AAV, especially in patients with MPO-ANCA, was reported in Japan ${ }^{34}$. A single nucleotide polymorphism in the promoter region of $M U C 5 B$ (rs35705950 (G/T)) encoding mucin $5 \mathrm{~B}$ had been considered to be a strong genetic factor in the pathogenesis of IPF. The risk allele (T) is associated with the overexpression of mucin $5 \mathrm{~B}$ in the lung, which results in pulmonary fibrosis ${ }^{35}$. Like IPF, rs35705950T was associated with ILD in MPO-ANCA-positive AAV patients. This association was even greater when confined to $\mathrm{AAV}$ patients with the UIP pattern. Indeed, most ILD patients with MPA exhibit the UIP pattern as seen in patients with IPF and rheumatoid arthritis.

\section{Pulmonary function tests}

In most patients with MPA and ILD, lung volumes are reduced in a restrictive pattern. This appears in pulmonary function tests as a reduction in total lung capacity (TLC), forced vital capacity, and diffusing capacity of the lungs for carbon monoxide (DLCO) $)^{5,36}$. In a study of AAV patients with ILD, the mean TLC was $77 \%$, and mean DLCO 56\%, of that predicted ${ }^{37}$. Comarmond et al. ${ }^{9}$ found that TLC and DLCO at diagnosis were more severely reduced in patients with AAV and pulmonary fibrosis who died at the end of follow-up. Interestingly, one third of MPA patients with ILD had a co-existing airflow obstruction ${ }^{7}$.

\section{Imaging}

The high-resolution computed tomography findings of ILD in MPA patients include ground-glass opacities (23\%-94\%), reticular opacities (41\%-77\%), interlobular septal thickening (41\%-71\%), consolidations (23\%-78\%), and honeycombing $(23 \%-52 \%)^{7,38}$. Airway abnormalities also have been reported in $32 \%-55 \%$ of the patients in the form of bronchiolitis, bronchial wall thickening, and bronchiectasis ${ }^{10,38}$. Lung involvement is usually symmetrical (50\%-100\%) and mainly affects the periphery and lower lobes of the lungs. The American Thoracic Society/European Respiratory Society International Multidisciplinary Classification of the Idiopathic Interstitial Pneumonias $^{39,40}$ shows that the most common radiologic pattern was UIP (50\%-57\%) (Figure 1), followed by nonspecific interstitial pneumonia (7\%-31\%) (Figure 2) and desquamative interstitial pneumonia $(14 \%)^{7,11}$. Combined pulmonary fibrosis and emphysema has been reported in MPA patients and those who are MPO-ANCA-positive ${ }^{38,41,42}$. Finally, $4 \%-40 \%$ of the studied cases did not fit any specific CT pattern, because of the coexistence of different patterns in the same patient ${ }^{11}$.

\section{Histopathology}

The typical histopathologic findings of ILD in MPA include fibrosis of the alveolar walls and interstitium and honeycomb- 

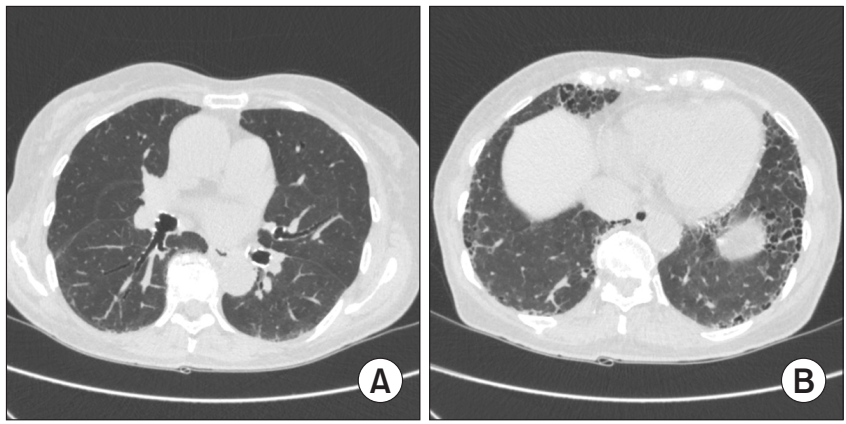

Figure 1. High-resolution computed tomography of usual interstitial pneumonia in a patient with microscopic polyangiitis. Mid(A) and lower (B) lung areas show peripheral reticulation, traction bronchiectasis, and honeycombing predominantly in the subpleural regions.

ing $^{14}$. The UIP pattern (patchy dense fibrosis with honeycomb changes, and actively fibrogenic fibroblastic foci) is the most predominant histopathologic pattern ${ }^{43,44}$. Prominent interstitial inflammation, the presence of lymphoid follicles, and small-airway involvement were more frequent in MPO-ANCApositive UIP than in idiopathic UIP ${ }^{11}$. Of note, the presence of active capillaritis or vasculitis has been rarely reported in ILD specimens with MPA ${ }^{45}$.

\section{Treatment}

Treatment for AAV should follow the international guidelines for vasculitis ${ }^{46}$. Although the treatment options of AAV are well-established, there is currently no specific recommendation for the treatment of ILD in AAV. In MPA patients with ILD, induction therapy usually includes systemic glucocorticoids $(88 \%-100 \%)$ in combination with cyclophosphamide (16\%-92\%) or rituximab (10\%) $)^{7,8,11}$. Azathioprine or mycophenolate mofetil are used as maintenance therapy for vasculitis. However, neither induction nor maintenance therapy has led to a full recovery of pulmonary function in most MPA patients with ILD $^{47}$.

Some studies have suggested that immunosuppressive therapy may be more effective in ANCA-positive ILD patients without vasculitis than in ANCA-negative patients ${ }^{23,45,48}$. Another study reported that a decline in pulmonary function and/or the progression of ILD prevailed despite immunosuppressive therapy ${ }^{7}$. Thus, general measures, such as supplemental oxygen, pulmonary rehabilitation, and proper vaccinations, are as important in patients with ANCA-positive ILD as in IPF management ${ }^{11}$. The radiographic findings of inflammatory changes, such as ground-glass opacity, tend to resolve completely after immunosuppressive treatment, whereas the chronic forms of lung fibrosis (i.e., honeycombing) usually remain unchanged. Immunosuppressive therapy also does not seem to reduce the risk of the subsequent development of

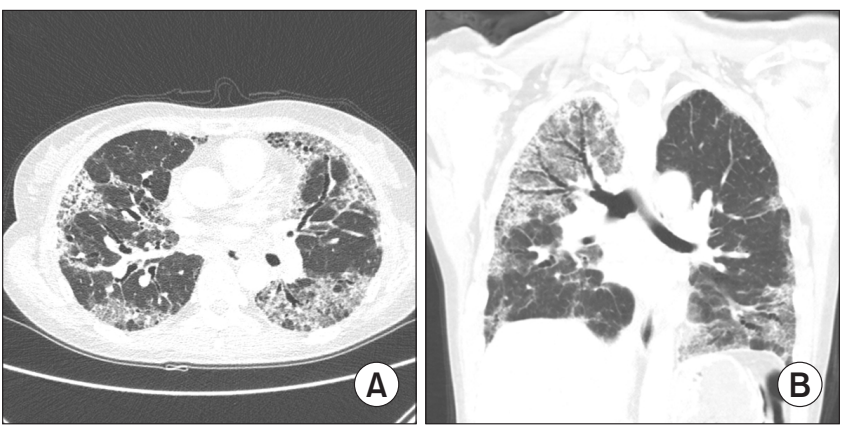

Figure 2. Axial (A) and coronal (B) high-resolution computed tomography scans in a patient with MPO-ANCA-positive nonspecific interstitial pneumonia. Radiographic findings show widespread diffuse patchy ground-glass opacities, reticular opacities, and traction bronchiectasis in both lungs. MPO: myeloperoxidase; ANCA: antineutrophil cytoplasmic antibody.

vasculitis.

Antifibrotic therapy (pirfenidone and nintedanib) could be considered for patients with ANCA-positive IPF according to the local and international treatment guidelines for $\mathrm{IPF}^{49}$. Furthermore, given both the fibrotic and the inflammatory aspects of AAV-ILD, the combination of immunosuppressive and antifibrotic therapy might be an opportunity for future therapeutic approaches. A clinical trial (NCT03385668) is currently ongoing to evaluate the efficacy and safety of the antifibrotic drug pirfenidone in patients with MPO-ANCA-positive pulmonary fibrosis with or without AAV.

\section{Prognosis}

ILD usually has a poor prognosis in MPA patients, because ILD frequently results in reduced lung function and chronic hypoxia. In previous studies, the death rate was 2 to 4 times higher in MPA patients with ILD than in those without ILD ${ }^{8,18}$. A previous study showed that one third of the MPA patients with ILD developed chronic respiratory insufficiency that required long-term oxygen therapy, and 31\%-85\% of the study patients died during the follow-up ${ }^{9}$. In other investigations, the 5 -year survival was only $19 \%-30 \%{ }^{7,18}$. The primary cause of death included the acute exacerbation of pulmonary fibrosis, sepsis, and progressive lung involvement, leading to end-stage respiratory failure $\mathrm{e}^{10,47}$.

The prognosis of ANCA-positive ILD patients without AAV is controversial. In patients with ILD and no systemic vasculitis, ANCA-positive and ANCA-negative patients had similar survival rates ${ }^{11,45}$. In contrast, in a large cohort of 504 patients with isolated pulmonary fibrosis, the 5- and 10-year death rates were significantly lower in ANCA-positive patients ${ }^{48}$. PR3-ANCA, age $>65$ years, a baseline DLCO of $<70 \%$, and a high titer of ANCA were associated with the death rate in ANCA-positive ILD ${ }^{48,50}$. 


\section{Diffuse Alveolar Hemorrhage}

DAH is defined as the accumulation of red blood cells within the alveolar space, derived from the alveolar capillaries or venules because of a disrupted alveolar-capillary interface ${ }^{51}$. It is estimated to occur in $10 \%$ to $47 \%$ of the patients with AAV and may be the initial presentation of $A A V^{51}$. The mean age at presentation was 57 years, with a similar gender prevalence. The most common clinical diagnosis accompanying DAH was MPA (52\%), followed by GPA (41\%), although the PR3ANCA positivity in this study was $57 \%^{52}$.

DAH is rarely asymptomatic in MPA. Most patients present with the rapid onset of dyspnea, overt hemoptysis, anemia, or progressive respiratory failure. It is associated with active rapid crescentic glomerulonephritis, which causes renal insufficiency, leading to pulmonary-renal syndrome ${ }^{53,54}$. Nonetheless, it may follow a more indolent course with recurrent hemoptysis. The symptoms can precede the diagnosis of DAH by more than a year in $25 \%$ of the cases ${ }^{55}$. DAH should be suspected in patients with respiratory symptoms, a falling hematocrit, radiographic abnormalities, including diffuse lung infiltrates and ground-glass opacities, and an elevated diffusing capacity.

\section{Pathogenesis}

DAH in AAV is a manifestation of immune-mediated pulmonary capillaritis. The activated neutrophils in the alveolar capillaries infiltrate the interstitium and undergo leukocytoclasis. With the accumulation of nuclear debris within the interstitium, the integrity of the alveolar capillary basement membranes is compromised. Some evidence suggests that ANCA plays a major part in the pathogenesis of DAH, but its exact role remains unknown. In an experimental murine model, the splenocytes of MPO-knockout mice immunized with MPO were injected into mice who lacked functioning $B$ and $T$ cells and who developed severe necrotizing glomerulonephritis and pulmonary capillaritis, which was augmented by the simultaneous injection of lipopolysaccharide. Furthermore, the alternative complement pathway has been demonstrated to be essential in developing the lesions. C5a is a powerful neutrophil chemoattractant and amplifies necrotizing inflammation of the vessel walls. Mice deficient in factor B or C5 of the complement system did not develop lesions ${ }^{56}$. However, this mechanism is yet unproven in humans.

\section{Imaging}

The common CT features of DAH are bilateral ground-glass opacity and alveolar consolidation with air bronchogram, but these are generally nonspecific ${ }^{57}$. The CT features are characteristically seen as patchy or diffuse, and are predominantly present in the upper and middle zones in two thirds of the cases. They are limited to the lower zones in a fourth of the $\operatorname{cases}^{55}$. The periphery of the lung is typically spared because of tapering of the pulmonary vasculature. With resorption of the hemorrhage, ill-defined centrilobular nodules may become visible because of the resolution of ground-glass opacities, and septal thickening may become visible ${ }^{57}$.

\section{Bronchoscopy and BAL}

Bronchoscopy helps to visualize fresh blood, and BAL fluid examination aids in the diagnosis of DAH by assessing its nature and identifying greater than $5 \%$ hemosiderin-laden macrophages [(hemosiderin-laden macrophages/total cells) $\times 100$ ] in the BAL fluid by Prussian blue staining. In addition, sequential progressive hemorrhagic BAL samples are indicative of $\mathrm{DAH}^{58}$. However, BAL fluid examination may show false negative results. Nevertheless, BAL helps to rule out other diagnoses, such as infection, and therefore is an important adjunct for the diagnosis of DAH.

\section{Histopathology}

Even though a lung biopsy is rarely performed ${ }^{59}$, the pulmonary histopathologic findings of DAH in AAV show necrotizing vasculitis of the small arteries, venules, and capillaries along with hemorrhage and scattered neutrophilic capillaritis. Capillaritis is identified by the thickening of the alveolar walls, which contain many neutrophils and nuclear dust. Immune deposits are absent in AAV, which differs from pulmonary capillaritis caused by systemic lupus erythematosus or antiglomerular basement membrane antibody disease. Of note, hemosiderin-laden macrophages appear in alveoli approximately 48 hours after the onset of a hemorrhage ${ }^{60}$.

\section{Treatment}

DAH in AAV requires pulses of intravenous glucocorticoids and cyclophosphamide. Rituximab is another option; its efficacy was as good as that of intravenous cyclophosphamide. The RAVE trial demonstrated similar remission rates at six months between the two groups ${ }^{61}$. Given previous small observational studies of AAV and anti-glomerular basement membrane antibody disease ${ }^{62,63}$, plasma exchange can be considered for the treatment of DAH in patients with AAV, yet its protocol and outcome remain controversial ${ }^{46}$. In the Plasma Exchange and Glucocorticoids for Treatment of ANCA-Associated Vasculitis (PEXIVAS) trial, where 191 among 704 patients with severe AAV were those with pulmonary hemorrhage, the addition of plasma exchange to immunosuppressive therapy did not reduce the incidence of death or end-stage kidney disease. These results were not different in the subgroup analysis according to the severity of pulmonary hemorrhage ${ }^{64}$. One meta-analysis also showed that plasma exchange was not associated with a lower death rate in patients with $\mathrm{AAV}^{65}$. 


\section{Prognosis}

DAH in AAV had an 8.6-fold increased relative risk of death ${ }^{66}$. However, Kostianovsky et al..$^{53}$ reported that DAH alone was not responsible for the increased death rate. They found that renal impairment associated with DAH was a predictor of late morbidity and death ${ }^{53}$. The incidence of recurrent DAH is relatively low, ranging from 10 to $31 \%^{53,55}$. Further studies are needed to distinguish the clinical features and outcomes of DAH in specific ANCA and AAV subsets ${ }^{67}$.

\section{Conclusion}

Although MPA is well-known for its clinical manifestation of necrotizing glomerulonephritis and DAH, ILD is now considered to be a common and serious pulmonary complication of MPA, especially in patients with MPO-ANCA. ILD can initially present as isolated pulmonary fibrosis preceding the diagnosis of MPA. ANCA should be tested for in all patients with isolated pulmonary fibrosis at diagnosis and during the course of the disease, because ANCA may have a significant prognostic effect on both survival and the risk of developing AAV, especially MPA. The pathogenic role of ANCA in the development of ILD and its association with histological phenotypes need further investigation. MPA patients with ILD, more than those without ILD, need special care when administered immunosuppressive therapy because of the higher risk of infection, such as pneumonia. Last, the optimal treatment strategies and key prognostic factors for ILD in MPA are yet to be established.

\section{Authors' Contributions}

Conceptualization: Shin K. Methodology and formal analysis: Kim MJ, Shin K. Writing - original draft preparation: Kim MJ. Writing - review and editing: Kim MJ, Shin K. Approval of final manuscript: all authors.

\section{Conflicts of Interest}

No potential conflict of interest relevant to this article was reported.

\section{Funding}

No funding to declare.

\section{References}

1. Bloch DA, Michel BA, Hunder GG, McShane DJ, Arend WP, Calabrese LH, et al. The American College of Rheumatology 1990 criteria for the classification of vasculitis: patients and methods. Arthritis Rheum 1990;33:1068-73.

2. Jennette JC, Falk RJ, Bacon PA, Basu N, Cid MC, Ferrario F, et al. 2012 revised International Chapel Hill Consensus Conference Nomenclature of Vasculitides. Arthritis Rheum 2013;65:1-11.

3. Mukhtyar C, Flossmann O, Hellmich B, Bacon P, Cid M, Cohen-Tervaert JW, et al. Outcomes from studies of antineutrophil cytoplasm antibody associated vasculitis: a systematic review by the European League Against Rheumatism systemic vasculitis task force. Ann Rheum Dis 2008;67:1004-10.

4. Lane SE, Watts RA, Shepstone L, Scott DG. Primary systemic vasculitis: clinical features and mortality. QJM 2005;98:97-111.

5. Huang H, Wang YX, Jiang CG, Liu J, Li J, Xu K, et al. A retrospective study of microscopic polyangiitis patients presenting with pulmonary fibrosis in China. BMC Pulm Med 2014;14:8.

6. Casian A, Jayne D. Management of alveolar hemorrhage in lung vasculitides. Semin Respir Crit Care Med 2011;32:33545.

7. Arulkumaran N, Periselneris N, Gaskin G, Strickland N, Ind PW, Pusey CD, et al. Interstitial lung disease and ANCA-associated vasculitis: a retrospective observational cohort study. Rheumatology (Oxford) 2011;50:2035-43.

8. Schirmer JH, Wright MN, Vonthein R, Herrmann K, Nolle B, Both $\mathrm{M}$, et al. Clinical presentation and long-term outcome of 144 patients with microscopic polyangiitis in a monocentric German cohort. Rheumatology (Oxford) 2016;55:71-9.

9. Comarmond C, Crestani B, Tazi A, Hervier B, Adam-Marchand $\mathrm{S}$, Nunes $\mathrm{H}$, et al. Pulmonary fibrosis in antineutrophil cytoplasmic antibodies (ANCA)-associated vasculitis: a series of 49 patients and review of the literature. Medicine (Baltimore) 2014;93:340-9.

10. Tzelepis GE, Kokosi M, Tzioufas A, Toya SP, Boki KA, Zormpala A, et al. Prevalence and outcome of pulmonary fibrosis in microscopic polyangiitis. Eur Respir J 2010;36:116-21.

11. Alba MA, Flores-Suarez LF, Henderson AG, Xiao H, Hu P, Nachman PH, et al. Interstital lung disease in ANCA vasculitis. Autoimmun Rev 2017;16:722-9.

12. Hozumi H, Enomoto N, Oyama Y, Kono M, Fujisawa T, Inui $\mathrm{N}$, et al. Clinical implication of proteinase-3-antineutrophil cytoplasmic antibody in patients with idiopathic interstitial pneumonias. Lung 2016;194:235-42.

13. Lionaki S, Blyth ER, Hogan SL, Hu Y, Senior BA, Jennette CE, et al. Classification of antineutrophil cytoplasmic autoantibody vasculitides: the role of antineutrophil cytoplasmic autoantibody specificity for myeloperoxidase or proteinase 3 in disease recognition and prognosis. Arthritis Rheum 2012;64: $3452-62$

14. Katsumata Y, Kawaguchi Y, Yamanaka H. Interstitial lung dis- 
ease with ANCA-associated vasculitis. Clin Med Insights Circ Respir Pulm Med 2015;9:51-6.

15. Pearce FA, Craven A, Merkel PA, Luqmani RA, Watts RA. Global ethnic and geographic differences in the clinical presentations of anti-neutrophil cytoplasm antibody-associated vasculitis. Rheumatology (Oxford) 2017;56:1962-9.

16. Yoo BW, Song JJ, Park YB, Lee SW. Clinical features of Korean elderly patients with antineutrophil cytoplasmic antibodyassociated vasculitis. Korean J Intern Med 2021;36:731-41.

17. Ahn JK, Hwang JW, Lee J, Jeon CH, Cha HS, Koh EM. Clinical features and outcome of microscopic polyangiitis under a new consensus algorithm of ANCA-associated vasculitides in Korea. Rheumatol Int 2012;32:2979-86.

18. Flores-Suarez LF, Ruiz N, Saldarriaga Rivera LM, Pensado L. Reduced survival in microscopic polyangiitis patients with pulmonary fibrosis in a respiratory referral centre. Clin Rheumatol 2015;34:1653-4.

19. Sebastiani M, Manfredi A, Vacchi C, Cassone G, Faverio P, Cavazza A, et al. Epidemiology and management of interstitial lung disease in ANCA-associated vasculitis. Clin Exp Rheumatol 2020;38 Suppl 124:221-31.

20. Tashiro H, Takahashi K, Tanaka M, Komiya K, Nakamura T, Kimura S, et al. Characteristics and prognosis of microscopic polyangiitis with bronchiectasis. J Thorac Dis 2017;9:303-9.

21. Oh JS, Lee CK, Kim YG, Nah SS, Moon HB, Yoo B. Clinical features and outcomes of microscopic polyangiitis in Korea. J Korean Med Sci 2009;24:269-74.

22. Solans-Laque R, Fraile G, Rodriguez-Carballeira M, Caminal L, Castillo MJ, Martinez-Valle F, et al. Clinical characteristics and outcome of Spanish patients with ANCA-associated vasculitides: Impact of the vasculitis type, ANCA specificity, and treatment on mortality and morbidity. Medicine (Baltimore) 2017;96:e6083.

23. Ando M, Miyazaki E, Ishii T, Mukai Y, Yamasue M, Fujisaki $\mathrm{H}$, et al. Incidence of myeloperoxidase anti-neutrophil cytoplasmic antibody positivity and microscopic polyangitis in the course of idiopathic pulmonary fibrosis. Respir Med 2013;107:608-15.

24. Idiopathic Pulmonary Fibrosis Clinical Research Network; Raghu G, Anstrom KJ, King TE Jr, Lasky JA, Martinez FJ. Prednisone, azathioprine, and $\mathrm{N}$-acetylcysteine for pulmonary fibrosis. N Engl J Med 2012;366:1968-77.

25. Chintagari NR, Jana S, Alayash AI. Oxidized ferric and ferryl forms of hemoglobin trigger mitochondrial dysfunction and injury in alveolar type I cells. Am J Respir Cell Mol Biol 2016;55:288-98.

26. Travis WD, Hoffman GS, Leavitt RY, Pass HI, Fauci AS. Surgical pathology of the lung in Wegener's granulomatosis: review of 87 open lung biopsies from 67 patients. Am J Surg Pathol 1991;15:315-33.

27. Schnabel A, Reuter M, Csernok E, Richter C, Gross WL. Subclinical alveolar bleeding in pulmonary vasculitides: correlation with indices of disease activity. Eur Respir J 1999;14:118-
24.

28. Guilpain P, Chereau C, Goulvestre C, Servettaz A, Montani D, Tamas N, et al. The oxidation induced by antimyeloperoxidase antibodies triggers fibrosis in microscopic polyangiitis. Eur Respir J 2011;37:1503-13.

29. Foucher P, Heeringa P, Petersen AH, Huitema MG, Brouwer E, Tervaert JW, et al. Antimyeloperoxidase-associated lung disease: an experimental model. Am J Respir Crit Care Med 1999;160:987-94.

30. Gehrig S, Duerr J, Weitnauer M, Wagner CJ, Graeber SY, Schatterny J, et al. Lack of neutrophil elastase reduces inflammation, mucus hypersecretion, and emphysema, but not mucus obstruction, in mice with cystic fibrosis-like lung disease. Am J Respir Crit Care Med 2014;189:1082-92.

31. Meyer KC, Raghu G, Baughman RP, Brown KK, Costabel U, du Bois RM, et al. An official American Thoracic Society clinical practice guideline: the clinical utility of bronchoalveolar lavage cellular analysis in interstitial lung disease. Am J Respir Crit Care Med 2012;185:1004-14.

32. Peterson MW, Monick M, Hunninghake GW. Prognostic role of eosinophils in pulmonary fibrosis. Chest 1987;92:51-6.

33. Yoshida M, Yamada M, Sudo Y, Kojima T, Tomiyasu T, Yoshikawa N, et al. Myeloperoxidase anti-neutrophil cytoplasmic antibody affinity is associated with the formation of neutrophil extracellular traps in the kidney and vasculitis activity in myeloperoxidase anti-neutrophil cytoplasmic antibodyassociated microscopic polyangiitis. Nephrology (Carlton) 2016;21:624-9.

34. Namba N, Kawasaki A, Sada KE, Hirano F, Kobayashi S, Yamada $\mathrm{H}$, et al. Association of MUC5B promoter polymorphism with interstitial lung disease in myeloperoxidase-antineutrophil cytoplasmic antibody-associated vasculitis. Ann Rheum Dis 2019;78:1144-6.

35. Seibold MA, Wise AL, Speer MC, Steele MP, Brown KK, Loyd JE, et al. A common $M U C 5 B$ promoter polymorphism and pulmonary fibrosis. N Engl J Med 2011;364:1503-12.

36. Fernandez Casares M, Gonzalez A, Fielli M, Caputo F, Bottinelli Y, Zamboni M. Microscopic polyangiitis associated with pulmonary fibrosis. Clin Rheumatol 2015;34:1273-7.

37. Maillet T, Goletto T, Beltramo G, Dupuy H, Jouneau S, Borie R, et al. Usual interstitial pneumonia in ANCA-associated vasculitis: a poor prognostic factor. J Autoimmun 2020;106:102338.

38. Yamagata M, Ikeda K, Tsushima K, Iesato K, Abe M, Ito T, et al. Prevalence and responsiveness to treatment of lung abnormalities on chest computed tomography in patients with microscopic polyangiitis: a multicenter, longitudinal, retrospective study of one hundred fifty consecutive hospitalbased Japanese patients. Arthritis Rheumatol 2016;68:713-23.

39. Travis WD, Costabel U, Hansell DM, King TE Jr, Lynch DA, Nicholson AG, et al. An official American Thoracic Society/ European Respiratory Society statement: update of the international multidisciplinary classification of the idiopathic interstitial pneumonias. Am J Respir Crit Care Med 
2013;188:733-48.

40. American Thoracic Society; European Respiratory Society. American Thoracic Society/European Respiratory Society International Multidisciplinary Consensus Classification of the Idiopathic Interstitial Pneumonias. This joint statement of the American Thoracic Society (ATS), and the European Respiratory Society (ERS) was adopted by the ATS board of directors, June 2001 and by the ERS Executive Committee, June 2001. Am J Respir Crit Care Med 2002;165:277-304.

41. Tzouvelekis A, Zacharis G, Oikonomou A, Koulelidis A, Steiropoulos $\mathrm{P}$, Froudarakis $\mathrm{M}$, et al. Combined pulmonary fibrosis and emphysema associated with microscopic polyangiitis. Eur Respir J 2012;40:505-7.

42. Tzouvelekis A, Zacharis G, Oikonomou A, Mikroulis D, Margaritopoulos G, Koutsopoulos A, et al. Increased incidence of autoimmune markers in patients with combined pulmonary fibrosis and emphysema. BMC Pulm Med 2013;13:31.

43. Baqir M, Yi EE, Colby TV, Cox CW, Ryu JH, Specks U. Radiologic and pathologic characteristics of myeloperoxidaseantineutrophil cytoplasmic antibody-associated interstitial lung disease: a retrospective analysis. Sarcoidosis Vasc Diffuse Lung Dis 2019;36:195-201.

44. Homma S, Matsushita H, Nakata K. Pulmonary fibrosis in myeloperoxidase antineutrophil cytoplasmic antibody-associated vasculitides. Respirology 2004;9:190-6.

45. Hosoda C, Baba T, Hagiwara E, Ito H, Matsuo N, Kitamura H, et al. Clinical features of usual interstitial pneumonia with anti-neutrophil cytoplasmic antibody in comparison with idiopathic pulmonary fibrosis. Respirology 2016;21:920-6.

46. Yates M, Watts RA, Bajema IM, Cid MC, Crestani B, Hauser T, et al. EULAR/ERA-EDTA recommendations for the management of ANCA-associated vasculitis. Ann Rheum Dis 2016;75:1583-94.

47. Hervier B, Pagnoux C, Agard C, Haroche J, Amoura Z, Guillevin L, et al. Pulmonary fibrosis associated with ANCA-positive vasculitides: retrospective study of 12 cases and review of the literature. Ann Rheum Dis 2009;68:404-7.

48. Kagiyama N, Takayanagi N, Kanauchi T, Ishiguro T, Yanagisawa T, Sugita Y. Antineutrophil cytoplasmic antibody-positive conversion and microscopic polyangiitis development in patients with idiopathic pulmonary fibrosis. BMJ Open Respir Res 2015;2:e000058.

49. Raghu G, Rochwerg B, Zhang Y, Garcia CA, Azuma A, Behr J, et al. An official ATS/ERS/JRS/ALAT clinical practice guideline: treatment of idiopathic pulmonary fibrosis. An update of the 2011 clinical practice guideline. Am J Respir Crit Care Med 2015;192:e3-19.

50. Nozu T, Kondo M, Suzuki K, Tamaoki J, Nagai A. A comparison of the clinical features of ANCA-positive and ANCAnegative idiopathic pulmonary fibrosis patients. Respiration 2009;77:407-15.

51. Nasser M, Cottin V. Alveolar hemorrhage in vasculitis (primary and secondary). Semin Respir Crit Care Med 2018;39:482-
93.

52. Gallagher H, Kwan JT, Jayne DR. Pulmonary renal syndrome: a 4-year, single-center experience. Am J Kidney Dis 2002;39:42-7.

53. Kostianovsky A, Hauser T, Pagnoux C, Cohen P, Daugas E, Mouthon L, et al. Alveolar haemorrhage in ANCA-associated vasculitides: 80 patients' features and prognostic factors. Clin Exp Rheumatol 2012;30(1 Suppl 70):S77-82.

54. Wilke L, Prince-Fiocco M, Fiocco GP. Microscopic polyangiitis: a large single-center series. J Clin Rheumatol 2014;20:179-82.

55. Lauque D, Cadranel J, Lazor R, Pourrat J, Ronco P, Guillevin L, et al. Microscopic polyangiitis with alveolar hemorrhage: a study of 29 cases and review of the literature. Groupe d'Etudes et de Recherche sur les Maladies "Orphelines" Pulmonaires (GERM”O”P). Medicine (Baltimore) 2000;79:222-33.

56. Xiao H, Heeringa P, Hu P, Liu Z, Zhao M, Aratani Y, et al. Antineutrophil cytoplasmic autoantibodies specific for myeloperoxidase cause glomerulonephritis and vasculitis in mice. J Clin Invest 2002;1 10:955-63.

57. Chung MP, Yi CA, Lee HY, Han J, Lee KS. Imaging of pulmonary vasculitis. Radiology 2010;255:322-41.

58. Lara AR, Schwarz MI. Diffuse alveolar hemorrhage. Chest 2010;137:1164-71.

59. Quadrelli S, Dubinsky D, Solis M, Yucra D, Hernandez M, Karlen H, et al. Immune diffuse alveolar hemorrhage: clinical presentation and outcome. Respir Med 2017;129:59-62.

60. Churg AM, Myers JL, Tazelaar HD, Wright JL. Thurlbeck's pathology of the lung. New York: Thieme; 2011.

61. Stone JH, Merkel PA, Spiera R, Seo P, Langford CA, Hoffman GS, et al. Rituximab versus cyclophosphamide for ANCAassociated vasculitis. N Engl J Med 2010;363:221-32.

62. Klemmer PJ, Chalermskulrat W, Reif MS, Hogan SL, Henke DC, Falk RJ. Plasmapheresis therapy for diffuse alveolar hemorrhage in patients with small-vessel vasculitis. Am J Kidney Dis 2003;42:1149-53.

63. Levy JB, Turner AN, Rees AJ, Pusey CD. Long-term outcome of anti-glomerular basement membrane antibody disease treated with plasma exchange and immunosuppression. Ann Intern Med 2001;134:1033-42.

64. Walsh M, Merkel PA, Peh CA, Szpirt WM, Puechal X, Fujimoto $\mathrm{S}$, et al. Plasma exchange and glucocorticoids in severe ANCA-associated vasculitis. N Engl J Med 2020;382:622-31.

65. Bellos I, Michelakis I, Nikolopoulos D. The role of plasma exchange in antineutrophil cytoplasmic antibody-associated vasculitis: a meta-analysis. Clin Rheumatol 2021;40:1447-56.

66. Haworth SJ, Savage CO, Carr D, Hughes JM, Rees AJ. Pulmonary haemorrhage complicating Wegener's granulomatosis and microscopic polyarteritis. Br Med J (Clin Res Ed) 1985;290:1775-8.

67. West S, Arulkumaran N, Ind PW, Pusey CD. Diffuse alveolar haemorrhage in ANCA-associated vasculitis. Intern Med 2013;52:5-13. 